\title{
A Unified Approach to Several Results Involving Integrals of Multifunctions (revision)
}

\author{
Erik J. Balder \\ Mathematical Institute, University of Utrecht \\ Utrecht, the Netherlands
}

\begin{abstract}
A well-known equivalence of randomization result of Wald and Wolfowitz states that any Young measure can be regarded as a probability measure on the set of all measurable functions. Here we give a sufficient condition for the Young measure to be equivalent to a probability measure on the set of all integrable selectors of a given multifunction. In this way, Aumann's identity for integrals of multifunctions can be interpreted in a novel fashion. By additionally applying a fundamental result from Young measure theory to uniformly $L_{1}$-bounded sequences of functions, Fatou's lemma in several dimensions, which is formulated in terms of the integral of a Kuratowski limes superior multifunction, can be proven in a new fashion. Also, a natural extension of these arguments leads to a generalization of a recent result by Artstein and Rzežuchowski [3].
\end{abstract}

\section{Introduction}

This paper further extends the range of applicability of the theory of weak convergence of Young measures, a subject initiated by Berliocchi-Lasry [12], and developed in [6, 7, 9, 10, 23]. The main objective is to demonstrate the use that can be made of an equivalence of randomization result for Young measures, due to Wald and Wolfowitz [26]. By such equivalence any Young measure going into (probability measures on) $\mathbb{R}^{d}$ can be seen as a probability measure on the set $\mathcal{L}_{\mathbb{R}^{d}}^{0}$ of all measurable functions going into $\mathbb{R}^{d}$. This result has been of importance for statistical decision theory and game theory $[26,14]$. The present context gives a simple sufficient condition for the Young measure so that the equivalent probability measure is carried by the subset $\mathcal{L}_{F}^{1}$ of $\mathcal{L}_{\mathbb{R}^{d}}^{0}$, consisting of all integrable selectors of a given multifunction $F$. When this result is applied "barycentrically" to a suitable Young measure, it causes Aumann's well-known identity [5] for the convex hull of the integral of the multifunction $F$ to follow by a well-known fact from probability theory: A vectorvalued random variable which takes its values in a convex set, has its expectation belong to that same set. A slight generalization of Aumann's result is also obtained in this way. For Fatou's lemma in several dimensions, studied by several authors (e.g., see $[21,16,2,19]$ ), a similar argument turns out to hold. The most general Fatou lemma of this kind was given in $[6,7]$; the present approach provides a proof which differs substantially from the one given there. Since Fatou's lemma in several dimensions deals with the integral of a Kuratowski limes superior multifunction $L$, verification of the above sufficiency condition, with its need for a suitably chosen Young measure, is a much more delicate question. Fortunately, a Prohorov-type theorem from Young measure theory [7], when applied to uniformly $L_{1}$-bounded sequences in $\mathcal{L}_{\mathbb{R}^{d}}^{1}$, instantly provides the existence of a Young measure of the desired kind. A related result of Artstein and Rzežuchowski [3] can be generalized by means of an extension of this approach: As in the case of the Fatou lemma, the Young measure associated to the uniformly $L_{1}$-bounded sequence is regarded as a probability measure on $\mathcal{L}_{L}^{1}$, the space of integrable selectors of the multifunction $L$. However, this time the following well-known result from probability theory must be invoked: A vector-valued random variable, which has its values in a convex set and its expectation in an extreme point of that same convex set, must be degenerate (i.e., a constant almost surely). 
We observe that, ever since the work of E.J. McShane, barycentric arguments for Young measures have played a role in the calculus of variations and "unilateral analysis". This paper shows that there is another, more globally oriented way to work with barycentric notions associated to Young measures, which had been left unexplored until now.

\section{Fundamental results}

Let $(\Omega, \mathcal{F}, \mu)$ be a finite measure space; let $\mathcal{L}_{\mathbb{R}^{d}}^{0}\left[\mathcal{L}_{\mathbb{R}^{d}}^{1}\right]$ be the prequotient space of all measurable [integrable] functions $f: \Omega \rightarrow \mathbb{R}^{d}$ from $(\Omega, \mathcal{F}, \mu)$ into $\left(\mathbb{R}^{d}, \mathbf{B}^{d}\right){ }^{1}$ A seminal idea, going back to L.C. Young, is as follows: any function $f \in \mathcal{L}_{\mathbb{R}^{d}}^{0}$ may be identified with its relaxation $\epsilon_{f}$, which is given by $\epsilon_{f}: \omega \mapsto[$ Dirac measure at $f(\omega)]$. It is easy to see that this device embeds $\mathcal{L}_{\mathbb{R}^{d}}^{0}$ into the set of transition probabilities (cf. [18, III.2]) from $(\Omega, \mathcal{F})$ into $\left(\mathbb{R}^{d}, \mathbf{B}^{d}\right)$. This set is denoted by $\mathcal{R}$. To stress Young's point of viewing transition probabilities as "generalized functions", the elements of $\mathcal{R}$ will also be called Young measures; in statistical decision theory they are sometimes called behavioral decision rules $[14,26]$.

Recall the following definition [13]: a normal integrand on $\Omega \times \mathbb{R}^{d}$ is a $\mathcal{F} \times \mathbf{B}^{d}$-measurable function $g: \Omega \times \mathbb{R}^{d} \rightarrow(-\infty,+\infty]$ such that $g(\omega, \cdot)$ is lower semicontinuous for every $\omega \in \Omega$. Let $\mathcal{G}$ denote the set of all such normal integrands. For each nonnegative normal integrand $g \in \mathcal{G}$ a functional $I_{g}: \mathcal{R} \rightarrow[0,+\infty]$ is defined by

$$
I_{g}(\delta):=\int_{\Omega}\left[\int_{\mathbb{R}^{d}} g(\omega, x) \delta(\omega)(d x)\right] \mu(d \omega)
$$

etc. (see [18, III.2] for details on such integrals). In particular, for the degenerate Young measure $\epsilon_{f}$ this gives

$$
I_{g}\left(\epsilon_{f}\right)=J_{g}(f):=\int_{\Omega} g(\omega, f(\omega)) \mu(d \omega)
$$

for every measurable function $f \in \mathcal{L}_{\mathbb{R}^{d}}^{0}$. Let $\theta$ be the $\sigma$-algebra on the set $\mathcal{L}_{\mathbb{R}^{d}}^{0}$ of all measurable functions, which is generated by the functionals $J_{g}$, for all nonnegative normal integrands $g \in \mathcal{G}$.

Let $F: \Omega \rightarrow 2^{\mathbb{R}^{d}}$ be a given multifunction with a $\mathcal{F} \times \mathbf{B}^{d}$-measurable graph and closed values. Recall that a function $f \in \mathcal{L}_{\mathbb{R}^{d}}^{0}$ is said to be a measurable selector [integrable selector] of $F$ if $f$ itself is measurable [integrable] and satisfies $f(\omega) \in F(\omega)$ for $\mu$-almost every $\omega$. The set of all such measurable [integrable] selectors of $F$ is denoted by $\mathcal{L}_{F}^{0}$ [respectively $\mathcal{L}_{F}^{1}$ ]. Corresponding to $F$, two special normal integrands $\chi_{F}$ and $g_{F}$ in $\mathcal{G}$ are defined as follows:

$$
g_{F}(\omega, x):= \begin{cases}|x| & \text { if } x \in F(\omega) \\ +\infty & \text { otherwise }\end{cases}
$$

The integrand $\chi_{F}$ is defined similarly, by replacing $|x|$ in the above expression by 0 ; it is what is know as the characteristic function of the multifunction $F$. Now observe that $\mathcal{L}_{F}^{0}\left[\mathcal{L}_{F}^{1}\right]$ consists precisely of all measurable functions $f \in \mathcal{L}_{\mathbb{R}^{d}}^{0}$ which satisfy $J_{\chi_{F}}(f)<+\infty$ [respectively, $J_{g_{F}}(f)<+\infty$ ]. Thus, it follows from the definition of the $\sigma$-algebra $\theta$ that $\mathcal{L}_{F}^{0}$ and $\mathcal{L}_{F}^{1}$ are $\theta$-measurable in $\mathcal{L}_{\mathbb{R}^{d}}^{0}$. Following Aumann [5], the integral of the multifunction $F$ is defined by

$$
\int_{\Omega} F d \mu:=\left\{\int_{\Omega} f d \mu: f \in \mathcal{L}_{F}^{1}\right\}
$$

Hence, $\int_{\Omega} F d \mu$ is the image of $\mathcal{L}_{F}^{1}$ under $\phi \mapsto \int_{\Omega} \phi d \mu$; note for future use that this mapping is $\theta$-measurable, as is evident from writing $\int \phi^{i} d \mu=\int \max \left(\phi^{i}, 0\right) d \mu-\int \max \left(-\phi^{i}, 0\right) d \mu, i=1, \cdots, d$. We now formulate a fundamental result, providing a sufficient condition for a Young measure in order to be representable as a probability measure on the set of all measurable [integrable] selectors of $F$.

\footnotetext{
${ }^{1} \mathbf{B}^{d}$ stands for the Borel $\sigma$-algebra on $\mathbb{R}^{d}$.
} 
Theorem 2.1 To every transition probability $\delta \in \mathcal{R}$ there corresponds a probability measure $\pi_{\delta}$ on the space $\mathcal{L}_{\mathbb{R}^{d}}^{0}$ of all measurable functions with $\sigma$-algebra $\theta$ such that

$$
I_{g}(\delta)=\int_{\mathcal{L}_{\mathbb{R}^{d}}^{0}} J_{g}(\phi) \pi_{\delta}(d \phi)
$$

for every nonnegative normal integrand $g \in \mathcal{G}$. Furthermore, if for almost every $\omega$ the measure $\delta(\omega)$ is carried by the set $F(\omega)$ (in other words, if $I_{\chi_{F}}(\delta)<+\infty$ ), then

$$
\pi_{\delta} \text { is carried by the set } \mathcal{L}_{F}^{0} \text { of all measurable selectors of } F .
$$

If moreover $I_{g_{F}}(\delta)<+\infty$, then

$$
\pi_{\delta} \text { is carried by the set } \mathcal{L}_{F}^{1} \text { of all integrable selectors of } F \text {. }
$$

Proof. We start by applying an extended version, as obtained in [17, Theorem 1], of the original equivalence result of Wald and Wolfowitz in [26]. Let $\theta^{\prime}$ denote the $\sigma$-algebra on $\mathcal{L}_{\mathbb{R}^{d}}^{0}$ generated by all functionals $f \mapsto \int_{\Omega} h(f(\omega)) P(d \omega)$, for all $h: \mathbb{R}^{d} \rightarrow \mathbb{R}$ bounded and $\mathbf{B}^{d}$-measurable, and all probability measures $P$ on $(\Omega, \mathcal{F})$. By [17, Theorem 1] there exists a probability measure $\pi_{\delta}$ on $\left(\mathcal{L}_{\mathbb{R}^{d}}^{0}, \theta^{\prime}\right)$ such that

$$
\int_{\Omega}\left[\int_{\mathbb{R}^{d}} h(x) \delta(\omega)(d x)\right] P(d \omega)=\int_{\mathcal{L}_{\mathbb{R}^{d}}^{0}}\left[\int_{\Omega} h(\phi(\omega)) P(d \omega)\right] \pi_{\delta}(d \phi)
$$

for all bounded $\mathbf{B}^{d}$-measurable functions $h: \mathbb{R}^{d} \rightarrow \mathbb{R}$ and all probability measures $P$ on $(\Omega, \mathcal{F})$. For the absolutely continuous probability measures given by $d P:=\psi d \mu / \int \psi d \mu$, with $\psi$ a nonnegative $\mu$-integrable function in $\mathcal{L}_{\mathbb{R}}^{1}$, the above identity gives that (1) holds for all $g(\omega, x)$ of the form $g(\omega, x):=h(x) \psi(\omega)$, with $h$ as above. Taking in particular $h$ to be bounded and continuous and using the approximation results in the proofs of Theorems 2.2, 2.1 in [9, pp. 268-269], it is then a small matter to deduce that $\theta^{\prime}$ contains $\theta$ and to establish the validity of (1) for all nonnegative normal integrands $g$ 's in $\mathcal{G}$.

Application of (1) with $g:=\chi_{F}$ gives $0=\int J_{\chi_{F}} d \pi_{\delta}=0$. Of course, this implies that for $\pi_{\delta^{-}}$almost every $\phi$ the identity $J_{\chi_{F}}(\phi)=0$ must hold. But this identity is equivalent to having $\phi$ in $\mathcal{L}_{F}^{0}$. This proves the first support statement about $\pi_{\delta}$.

The second support statement follows in the same way, this time by using $g:=g_{F}$ in (1). Q.E.D.

Recall that the barycenter of a probability measure $\nu$ on $\left(\mathbb{R}^{d}, \mathbf{B}^{d}\right)$ is defined by

$$
\text { bar } \nu:=\int_{\mathbb{R}^{d}} x \nu(d x)
$$

provided that it exists, i.e., provided that $\int_{\mathbb{R}^{d}}|x| \nu(d x)<+\infty$. Further, the convex hull of a set $D \subset \mathbb{R}^{d}$ is denoted by co $D$, as usual.

Theorem 2.2 Suppose that the Young measure $\delta \in \mathcal{R}$ satisfies $I_{g_{F}}(\delta)<+\infty$. Then

$$
\int_{\Omega} \operatorname{bar} \delta(\omega) \mu(d \omega) \in \operatorname{co} \int_{\Omega} F d \mu
$$

If also

$$
\int_{\Omega} \operatorname{bar} \delta(\omega) \mu(d \omega) \text { is an extreme point of co } \int_{\Omega} F d \mu
$$

then there exists an essentially unique $f \in \mathcal{L}_{F}^{1}$ such that

$$
\delta(\omega)=\epsilon_{f}(\omega) \text { a.e. }
$$


Proof. As a first consequence of the finiteness hypothesis, we have

$$
\int_{\Omega} \int_{\mathbb{R}^{d}}|x| \delta(\omega)(d x) \mu(d \omega)<+\infty .
$$

This immediately implies the existence a.e. and integrability over $\Omega$ of bar $\delta(\omega)$. Let $\pi_{\delta}$ be the probability measure on $\left(\mathcal{L}_{\mathbb{R}^{d}}^{0}, \theta\right)$, as asserted in Theorem 2.1. We now apply (1) to the normal integrands $g_{i}, g_{d+i} \in \mathcal{G}, i=1, \cdots, d$, given by $g_{i}(\omega, x):=\max \left(x^{i}, 0\right)$ and $g_{d+i}(\omega, x):=\max \left(-x^{i}, 0\right)$. Subtraction and vectorization give

$$
a:=\int_{\Omega} \operatorname{bar} \delta d \mu=\int_{\mathcal{L}_{F}^{1}}\left[\int_{\Omega} \phi d \mu\right] \pi_{\delta}(d \phi) .
$$

Define the random variable $\iota$ on $\left(\mathcal{L}_{F}^{1}, \theta, \pi_{\delta}\right)$ by setting $\iota(\phi):=\int \phi d \mu$. By a classical result (see [14, p. 74] and [20]) the point $a$, which is the expectation of $\iota$ by the above formula, must belong to the convex hull of the set $\iota\left(\mathcal{L}_{F}^{1}\right)$ (thanks to [20] we need not even ensure measurability of the that set). But by definition of $\iota$, that set is precisely the Aumann integral $\int F d \mu$. Clearly, this settles the first statement.

In view of the above, the extremality hypothesis for the second statement implies that $a$, the expectation of $\iota$, is an extreme point of $\iota\left(\mathcal{L}_{F}^{1}\right)$. It is easy to see that this implies a.e.-constancy of the random variable $\iota$ on $\left(\mathcal{L}_{F}^{1}, \theta, \pi_{\delta}\right)$ (e.g., see the proof of [8, Lemma 3$]$ ). Therefore, $\pi_{\delta}$ is carried by $\iota^{-1}(a)$. Now note that the extremality of $a$ implies that $a$ must belong to the set $\int F d \mu$ itself (not just to its convex hull). So there exists $f \in \mathcal{L}_{F}^{1}$ such that $a=\int_{\Omega} f d \mu$. If one has also $a=\int_{\Omega} f^{\prime} d \mu$ for some other $f^{\prime} \in \mathcal{L}_{F}^{1}$, then $\int_{B}\left(f^{\prime}-f\right) d \mu=0$ for every $B \in \mathcal{F}$, or else a contradiction with a's extremality would result. This argument shows that there is an essentially unique $f$ satisfying $a=\int f d \mu=\iota(f)$. So $\iota^{-1}(a)$, which carries $\pi_{\delta}$, consists of an essentially unique $f \in \mathcal{L}_{F}^{1}$. By $(1)$ this implies that $I_{g}(\delta)=J_{g}(f)=I_{g}\left(\epsilon_{f}\right)$ for all nonnegative normal integrands $g$. Taking special $g$ 's of the form $g(\omega, x)=\psi(\omega) h(x)$ used in the proof of Theorem 2.1, it is easy to deduce that $\delta(\omega)=\epsilon_{f}(\omega)$ for a.e. $\omega$ (use the fact that the set of all continuous and bounded such $h$ 's has a countable subset which separates any two probability measures on $\mathbb{R}^{d}$ ). Q.E.D.

Remark 2.3 Note that in Theorem 2.2

$$
\int_{\Omega} \operatorname{bar} \delta(\omega) \mu(d \omega) \in \operatorname{co} \int_{\Omega} F d \mu \subset \int_{\Omega} \operatorname{co} F d \mu .
$$

Here co $F: \Omega \rightarrow 2^{\mathbb{R}^{d}}$ is defined by taking pointwise convex hulls of the sets $F(\omega)$. It is important to realize that the inclusion can be strict (see for instance [25]). If one were to know in advance that Aumann's identity (see Proposition 3.1 below) happened to hold here, then an alternative, much simpler proof of Theorem 2.2 could be given, based on the fact that $\delta(\omega)(F(\omega))=1$ a.e. For this gives bar $\delta(\omega) \in \operatorname{co} F(\omega)$ a.e., by pointwise application of the cited result from [14, 20$]$.

Remark 2.4 The case where $F$ has closed values but a nonmeasurable graph can be covered very simply by the results in this section. Namely, by [1, Theorem 2.7] there exists a countable collection $\left(\bar{f}_{j}\right) \subset \mathcal{L}_{F}^{0}$ such that for every $\phi \in \mathcal{L}_{F}^{0}$ one has $\phi(\omega) \in \bar{F}(\omega):=\operatorname{cl}\left(\bar{f}_{j}(\omega)\right)$ a.e. Of course, $\bar{F}$ is a closed-valued multifunction with measurable graph, and one can apply what was found previously, since $\mathcal{L}_{\bar{F}}^{p}=\mathcal{L}_{F}^{p}, p=0,1$.

\section{Applications}

Let us begin by proving that Aumann's well-known theorem on the integral of a multifunction [5] follows from Theorem 2.2. As in the previous section, $F: \Omega \rightarrow 2^{\mathbb{R}^{d}}$ is a multifunction with measurable graph and closed values. 
Proposition 3.1 (Aumann's identity [5]) Suppose that $F$ is nonnegative; that is, suppose that $F(\omega) \subset \mathbb{R}_{+}^{d}$ for almost all $\omega$. Then

$$
\operatorname{co~} \int_{\Omega} F d \mu=\int_{\Omega} \operatorname{co} F d \mu
$$

Proof. Obviously, the left side is contained in the right side. Conversely, let $f$ be an arbitrary function in $\mathcal{L}_{\mathrm{CO} F}^{1}$. By Carathéodory's theorem and an easy measurable selection argument [13, p. 101], there exist measurable selectors $f_{1}, \cdots, f_{d+1} \in \mathcal{L}_{F}^{0}$ and measurable functions $\alpha_{1}, \cdots, \alpha_{d+1}$ : $\Omega \rightarrow[0,1], \sum_{i} \alpha_{i}=1$, such that $f(\omega)=\sum_{i} \alpha_{i}(\omega) f_{i}(\omega)$ a.e. Define the transition probability $\delta_{f} \in \mathcal{R}$ by $\delta_{f}(\omega)=\sum_{i} \alpha_{i}(\omega) \epsilon_{f_{i}}(\omega)$. Then $I_{g_{F}}\left(\delta_{f}\right)=\int_{\Omega} \sum_{i} \alpha_{i}(\omega)\left|f_{i}(\omega)\right| \mu(d \omega)<+\infty$, as follows directly from nonnegativity of the component functions of all $f_{i}$. Observe that bar $\delta_{f}(\omega)=\sum_{i} \alpha_{i}(\omega) f_{i}(\omega)=$ $f(\omega)$. So Theorem 2.2 gives that $\int_{\Omega} f d \mu$ belongs to the left side. Q.E.D.

A slight extension of Aumann's result can now also be formulated; it follows directly from the proof given above. Note that this result deals with measurable functions $f_{i}$ that need not be integrable.

Proposition 3.2 Suppose that $f \in \mathcal{L}_{\mathbb{R}^{d}}^{1}$ is such that $f(\omega)=\sum_{i} \alpha_{i}(\omega) f_{i}(\omega)$ a.e., with $f_{1}, \cdots, f_{m}$ in $\mathcal{L}_{\mathbb{R}^{d}}^{0}$ and $\alpha_{1}, \cdots, \alpha_{m}: \Omega \rightarrow[0,1]$ measurable, with $\sum_{i} \alpha_{i}=1$ a.e. If

$$
\int_{\Omega} \sum_{i=1}^{m} \alpha_{i}(\omega)\left|f_{i}(\omega)\right| \mu(d \omega)<+\infty
$$

then

$$
\int_{\Omega} f d \mu \in \operatorname{co} \int_{\Omega} F^{\prime} d \mu
$$

where $F^{\prime}(\omega):=\left\{f_{1}(\omega), \cdots, f_{m}(\omega)\right\}$.

It will now be shown how the unifying Fatou lemma in several dimensions $[6,7]$, stated in Proposition 3.5 below, can be obtained by combining Theorem 2.2 with the following fundamental result from Young measure theory. Recall that the symbol Ls $\left(x_{m}\right)$ commonly denotes the set of all limit points of a sequence $\left(x_{m}\right)$ in $\mathbb{R}^{d}$; i.e., this set is the limes superior of $\left(x_{m}\right)$ in the sense of Kuratowski [4]. Recall also [16] that the finite measure space $\Omega$ can always be decomposed into two disjoint parts: a purely atomic part $\Omega^{p a}$ and a nonatomic part $\Omega^{n a}$.

Theorem $3.3([7])$ Suppose that $\left(f_{k}\right) \subset \mathcal{L}_{\mathbb{R}^{d}}^{1}$ is such that

$$
\sup _{k} \int_{\Omega}\left|f_{k}\right| d \mu<+\infty
$$

Then there exist a subsequence $\left(f_{m}\right)$ of $\left(f_{k}\right)$ and a Young measure $\tilde{\delta} \in \mathcal{R}$ such that

(i) For a.e. $\omega$

$$
\tilde{\delta}(\omega)(L(\omega))=1,
$$

where $L(\omega):=\operatorname{Ls}\left(f_{m}(\omega)\right)$.

(ii) For every normal integrand $g \in \mathcal{G}$

$$
\liminf _{m} J_{g}\left(f_{m}\right) \geq I_{g}(\tilde{\delta}),
$$

provided that the sequence $\left(\min \left(g\left(\cdot, f_{k}(\cdot)\right), 0\right)\right)$ is uniformly integrable.

Moreover, the subsequence $\left(f_{m}\right)$ can always be chosen in such a way that

$L(\omega)$ is a singleton for a.e. $\omega \in \Omega^{p a}$. 
The above result follows immediately from substituting [7, Example 2.3] in the fundamental Prohorov-type theorem for Young measure theory [7, Theorem I]. Basically, this is a relative compactness criterion for narrow convergence of Young measures, and the uniform $L_{1}$-boundedness of $\left(f_{k}\right)$ implies tightness in the sense of [7]. The additional stipulation involving $\Omega^{p a}$ follows by a simple diagonal extraction argument; see [7, p. 575].

Corollary 3.4 In Theorem 3.9

$$
\int_{\Omega^{n a}} \text { bar } \tilde{\delta} d \mu \text { exists and belongs to } \int_{\Omega^{\text {na }}} L d \mu \text {. }
$$

Proof. Substituting $g(\omega, x):=|x|$ in $(2)$ and taking into account that $\tilde{\delta}(\omega)$ is carried by $L(\omega)$ gives

$$
I_{g_{L}}(\tilde{\delta}) \leq \liminf _{m} \int_{\Omega}\left|f_{m}\right| d \mu \leq \sup _{k} \int_{\Omega}\left|f_{k}\right| d \mu<+\infty .
$$

Now restrict this to $\Omega^{n a}$ and apply Theorem 2.2 with $F:=L$. Note finally that the set $\int_{\Omega^{n a}} L d \mu$ is convex by Richter's theorem [16]. Q.E.D.

Theorem 3.3 and Corollary 3.4 can now be used to derive the following result, which is in essence the unifying Fatou lemma of $[6,7]$, rewritten in terms of the cone $C$.

Proposition 3.5 Suppose that $\left(f_{k}\right) \subset \mathcal{L}_{\mathbb{R}^{d}}^{1}$ is such that

$$
\sup _{k} \int_{\Omega}\left|f_{k}\right| d \mu<+\infty
$$

and that

$$
a:=\lim _{k} \int_{\Omega} f_{k} d \mu \text { exists. }
$$

Let $C$ be the convex cone of all $y \in \mathbb{R}^{d}$ for which

$$
\left(\min \left(y \cdot f_{k}, 0\right)\right) \text { is uniformly integrable. }
$$

Then

$$
a \in \int_{\Omega} \operatorname{Ls}\left(f_{k}\right) d \mu-C^{0},
$$

where $C^{0}:=\left\{z \in \mathbb{R}^{d}: z \cdot y \leq 0\right.$ for all $\left.y \in C\right\}$ is the polar cone of $C$.

Proof. Let $\left(f_{m}\right)$ be the subsequence of $\left(f_{k}\right)$ in Theorem 3.3, with the associated Young measure $\tilde{\delta}$. Let $L$ be again the pointwise limes superior multifunction; define $f_{*}$ by setting $f_{*}(\omega):=$ the single point contained in the singeton $L(\omega)$ for a.e. $\omega$ in $\Omega^{p a}$ (cf. Theorem 3.3). Measurability and integrability of $f_{*}$ on $\Omega^{p a}$ follow almost trivially. Clearly, by (i) of Theorem 3.3 we find on $\Omega^{p a}$ that $\tilde{\delta}(\omega)=\epsilon_{f_{*}}(\omega)$ a.e. As for the nonatomic part, Corollary 3.4 gives

$$
b:=\int_{\Omega^{n a}} \operatorname{bar} \tilde{\delta} d \mu \in \int_{\Omega^{n a}} L d \mu .
$$

So there exists $f_{*} \in \mathcal{L}_{L}^{1}\left(\Omega^{n a}\right)$ such that $b=\int_{\Omega^{n a}} f_{*} d \mu$. For arbitrary $y \in C$ an application of (2) to $g(\omega, x):=y \cdot x$, gives

$$
y \cdot a \geq \int_{\Omega} y \cdot \operatorname{bar} \tilde{\delta}(\omega) \mu(d \omega)=y \cdot \int_{\Omega} f_{*} d \mu,
$$

by aggregating the above results. This proves the desired result. Q.E.D.

As explained in [6], Proposition 3.5 captures not only all known Fatou lemmas of this type (e.g. $[21,16,2]$ ), but also related variational existence results. Observe that the proofs given in $[6,7]$ differ notably from the approach followed here. See [11] for recent extensions, generated by Proposition 3.5, to multivalued functions $f_{k}$.

Next, another application will be presented which also combines Theorem 2.2 with Theorem 3.3. This generalizes a recent result of Artstein and Rzežuchowski [3] (the latter result is recalled in Corollary 3.7 below). 
Theorem 3.6 Suppose that $\left(f_{k}\right) \subset \mathcal{L}_{\mathbb{R}^{d}}^{1}$ is such that

$$
\sup _{k} \int_{\Omega}\left|f_{k}\right| d \mu<+\infty
$$

and that

$$
a:=\lim _{k} \int_{\Omega} f_{k} d \mu \text { exists. }
$$

Suppose further that

$$
a \text { is an extreme point of } \operatorname{co} \int_{\Omega} \operatorname{Ls}\left(f_{k}\right) d \mu
$$

and that a has the following maximality property with respect to the partial order induced by the polar $C^{0}$ of $C$ :

$$
\left(\operatorname{co} \int_{\Omega} \operatorname{Ls}\left(f_{k}\right) d \mu-a\right) \cap C^{0}=\{0\}
$$

as before, $C$ is the convex cone of all $y \in \mathbb{R}^{d}$ such that

$$
\left(\min \left(y \cdot f_{k}, 0\right)\right) \text { is uniformly integrable. }
$$

Then there exists an essentially unique $f_{*} \in \mathcal{L}_{\mathrm{Ls}\left(f_{k}\right)}^{1}$ such that

$$
\left(f_{k}\right) \text { converges in measure to } f_{*} \text { and } \int_{\Omega} f_{*} d \mu=a \text {. }
$$

Proof. Start out by taking an arbitrary subsequence from $\left(f_{k}\right)$. Exactly as in the proof of Proposition 3.5 one finds a Young measure $\tilde{\delta} \in \mathcal{R}$, associated with some subsequence $\left(f_{m}\right)$ of the subsequence already taken, for which (2) can be applied to $g(\omega, x):=y \cdot x, y \in C$. This gives $\int_{\Omega}$ bar $\tilde{\delta} d \mu-a \in C^{0}$. By the hypothesized intersection property, it follows that the equality $a=\int_{\Omega}$ bar $\tilde{\delta} d \mu$ must hold. So Theorem 2.2 may be invoked, and it follows that $\tilde{\delta}(\omega)=\epsilon_{f_{*}}(\omega)$ a.e. for an essentially unique function $f_{*} \in \mathcal{L}_{\mathrm{Ls}\left(f_{k}\right)}^{1}$. The essential part of this proof consists of applying (2) to $g(\omega, x):=-1$ if $\left|x-f_{*}(\omega)\right| \geq \epsilon$ and $g(\omega, x):=0$ otherwise, for $\epsilon>0$. This gives

$$
\liminf _{m} J_{g}\left(f_{m}\right)=-\limsup _{m} \mu\left(\left\{\omega \in \Omega:\left|f_{m}(\omega)-f_{*}(\omega)\right| \geq \epsilon\right\}\right) \geq J_{g}\left(f_{*}\right)=0 .
$$

Hence, $\left(f_{m}\right)$ converges in measure to $f_{*}$, a function which does not depend on the initial subsequence. So the conclusion is that every subsequence of $\left(f_{k}\right)$ has a subsequence converging in measure to the function $f_{*}$. This is equivalent to what had to be demonstrated. Q.E.D.

Corollary 3.7 ([3]) Suppose that $(\Omega, \mathcal{F}, \mu)$ is nonatomic. Suppose that $\left(f_{k}\right) \subset \mathcal{L}_{\mathbb{R}^{d}}^{1}$ is such that

$$
\left(f_{k}\right) \text { is uniformly integrable }
$$

and that

$$
a:=\lim _{k} \int_{\Omega} f_{k} d \mu \text { exists }
$$

with

$$
a \text { is an extreme point of } \int_{\Omega} \operatorname{Ls}\left(f_{k}\right) d \mu \text {. }
$$

Then there exists an essentially unique $f_{*} \in \mathcal{L}_{\mathbb{R}^{d}}^{1}$ such that

$$
\lim _{k \rightarrow \infty} \int_{\Omega}\left|f_{k}-f_{*}\right| d \mu=0
$$


Proof. Theorem 3.6 can be invoked, taking into consideration Richter's theorem [16]. Here $C=\mathbb{R}^{d}$, so $C^{0}=\{0\}$, and this makes the maximality property trivial. Of course, under the uniform integrability hypothesis, convergence in measure implies $L_{1}$-convergence [18, Proposition II.5.4]. Q.E.D.

Let us consider an example in which the conditions of Theorem 3.6 hold, but those of Corollary 3.7 do not:

Example 3.8 Equip $\Omega:=[0,1]$ with Lebesgue $\sigma$-algebra and measure, and take $d:=1$. Consider the sequence $\left(f_{k}\right)$ of functions given by $f_{k}:=k$ on $[0,1 / k], f_{k}:=-k$ on $(1 / k, 2 / k]$ and $f_{k}:=0$ elsewhere. Then $\left(f_{k}\right)$ is not uniformly integrable. Yet by $a=0$ and $\operatorname{Ls}\left(f_{k}(\omega)\right)=\{0\}$ all conditions of Theorem 3.6 are easily seen to hold.

Remark 3.9 It is interesting to observe that the result of Artstein and Rze zuachowski follows from Visintin's theorem as improved by the present author [24, 8]. This nicely complements their own observation [3, p. 93] that the converse implication is also true. Let the reasoning just be sketched (as in Corollary 3.7, suppose nonatomicity). Of course, there again exists an essentially unique $f_{*}$ in $\mathcal{L}_{\mathrm{Ls}\left(f_{k}\right)}^{1}$ such that $a=\int_{\Omega} f_{*} d \mu$. Thus, by a well-known property of extreme points, the extreme point condition for a in Corollary 3.7 implies that pointwise

$$
f_{*}(\omega) \text { is an extreme point of } \operatorname{co~} \operatorname{Ls}\left(f_{k}(\omega)\right) \text { a.e. }
$$

This is precisely the condition imposed in the extension of Visintin's theorem introduced in [8]. Let $\left(f_{n}\right)$ be an arbitrary subsequence of $\left(f_{k}\right)$. By the uniform integrability hypothesis it follows that a subsequence $\left(f_{m}\right)$ of $\left(f_{n}\right)$ converges weakly. Actually, $\left(f_{m}\right)$ has no choice but to converge weakly to $f_{*}$, by the essential uniqueness property of $f_{*}$. So the Visintin theorem of [8], applied to $\left(f_{m}\right)$, gives that $\left(f_{m}\right)$ also converges strongly to $f_{*}$. Since $\left(f_{n}\right)$ was arbitrary, this alternative proof of Corollary 3.7 is finished. Using the Gaposhkin biting lemma [15], a similar proof can also be given for Theorem 3.6.

Concluding remarks. i. Mention should be made of an interesting paper by Wagner [25], which gives necessary and sufficient conditions for Aumann's identity to hold without additional restrictions (but note that a significant part of the proof follows [5]). An open question is how to use this result so as to derive Proposition 3.2 more directly.

ii. M. Valadier has kindly pointed out to me that a condition quite similar to the one used in Proposition 3.2 occurs in [22, Theorem 4], and that it might be interesting to find a common link between his result - which deals with a kind of bang-bang approximation - and Proposition 3.2.

iii. Corollary 3.4 was derived by combining the Prohorov-type Theorem I of [7] with its Example 2.3. Similarly, a well-known lemma of Artstein [2, Lemma C] follows by combining Theorem I and Example 2.2 of [7], and using pointwise the barycentric result from [14, 20] (cf. Remark 2.3).

iv. Theorem 3.3 was also used to prove the unifying Fatou lemma in $[6,7]$, but in connection with an extreme point argument. Since Theorem 2.1 can also be proven by using Choquet integral representation theory, this forms the common link with the proof of Proposition 3.5 given here.

v. Artstein and Rzežuchowski prove their result in a very simple way by using the Fatou lemma of Artstein [2]. Parallel attempts to derive Theorem 3.6 equally simply from the unifying Fatou lemma would seem to fail because of the greater complexity of the problem being addressed.

vi. A well-known result of Olech [19] is rather similar to Theorem 3.6 and Corollary 3.7. The principal differences can be summarized as follows: Olech's result requires $a$ to be an extreme point of the closed convex hull of a set of type $\int_{\Omega} F d \mu$, but in return it does not require any uniform $L_{1}$-boundedness and, moreover, allows an analysis of the associated convergence rate [4, Theorem 8.6.3, Lemma 8.7.1].

vii. Partial extensions of some of the results in this paper to the infinite-dimensional case are possible, but these become arguably simpler. The reasons for this lie in the breakdown of Lyapunov's 
theorem in infinite dimensions, which makes the resulting mathematical compromises (in terms of closures of integrals of multifunctions) much easier to analyze; for this reason this subject has not been discussed here (see e.g. [11] for more information).

Acknowledgment. I am extremely grateful to an anonymous referee for many very perceptive comments about the style and presentation of an earlier version of this paper, and for generous suggestions on how to improve. Also, I wish to thank Michel Valadier for some helpful remarks.

\section{References}

[1] Artstein, Z. (1972). Weak convergence of set-valued functions and control. SIAM J. Control 13 $865-878$.

[2] Artstein, Z. (1979). A note on Fatou's lemma in several dimensions. J. Math. Econom. 6 277282.

[3] Artstein, Z. and Rzežuchowski, T. (1991). A note on Olech's lemma. Studia Math. 98 91-94.

[4] Aubin, J.-P. and Frankowska, H. (1990). Set-Valued Analysis. Birkhäuser, Boston.

[5] Aumann, R.J. (1965). Integrals of set-valued functions. J. Math. Anal. Appl. 12 1-12.

[6] Balder, E.J. (1984). A unifying note on Fatou's lemma in several dimensions. Math. Oper. Res. $9267-275$.

[7] Balder, E.J. (1984). A general approach to lower semicontinuity and lower closure in optimal control theory. SIAM J. Control Optim. 22 570-598.

[8] Balder, E.J. (1986). On weak convergence implying strong convergence in $L_{1}$-spaces. Bull. Austral. Math. Soc. 33 363-368.

[9] Balder, E.J. (1988). Generalized equilibrium results for games with incomplete information. Math. Oper. Res. 13 265-276.

[10] Balder, E.J. (1989). On Prohorov's theorem for transition probabilities. Travaux Sém. Anal. Convexe Montpellier 19 9.1-9.11.

[11] Balder, E.J. and Hess, C. (1993). Fatou's lemma for multifunctions with unbounded values. Math. Oper. Res., to appear.

[12] Berliocchi, H. and Lasry, J.-M. (1973). Intégrandes normales et mesures paramétrées en calcul des variations. Bull. Soc. Math. France 101 129-184.

[13] Castaing, C. and Valadier, M. (1977). Convex Analysis and Measurable Multifunctions. Lecture Notes in Mathematics 588. Springer-Verlag, Berlin.

[14] Ferguson, T.S. (1967). Mathematical Statistics: a Decision Theoretic Approach. Academic Press, New York.

[15] Gaposkhin, V.F. (1972). Convergence and limit theorems for sequences of random variables. Theory Prob. and Appl. 17 379-400.

[16] Hildenbrand, W. (1974). Core and Equilibria of a Large Economy. Princeton University Press, Princeton.

[17] Kirschner, H.-P. (1976). On a theorem of Wald and Wolfowitz on randomization in statistics. Manuscripta Math. 18 249-256.

[18] Neveu, J. (1964). Bases Mathématiques du Calcul des Probabilités. Masson, Paris. 
[19] Olech, C. (1976). Existence theory in optimal control, in : Control Theory and Topics in Functional Analysis. IAEA, Vienna, pp. 291-328.

[20] Pfanzagl, J. (1974). Convexity and conditional expectations. Annals Prob. 2 490-494.

[21] Schmeidler, D. (1970). Fatou's lemma in several dimensions. Proc. Amer. Math. Soc. 24 300306.

[22] Valadier, M. (1984). Some bang-bang theorems, in: Multifunctions and Integrands (G. Salinetti, ed.). Lecture Notes in Mathematics 1091. Springer-Verlag, Berlin, pp. 225-234.

[23] Valadier, M. (1990). Young measures, in: Methods of Nonconvex Analysis (A. Cellina, ed.). Lecture Notes in Mathematics 1446. Springer-Verlag, Berlin, pp. 152-188.

[24] Visintin, A. (1984). Strong convergence results related to strict convexity. Comm. Partial Differential Equations 9 439-466.

[25] Wagner, D.H. (1975). Integral of a convex-hull-valued function. J. Math. Anal. Appl.50 548559.

[26] Wald, A. and Wolfowitz, J. (1951). Two methods of randomization in statistics and the theory of games. Ann. Math. 53 581-586. 